\title{
Modeling the steering wheel influence by the driver on the vehicle's motion stability
}

\author{
Doniyor Akhmedov ${ }^{1}$, Shavkat Alimukhamedov ${ }^{2}$, Ibragim Tursunov $^{2}$, Soyib Narziev $^{2}$ and \\ Davron Riskaliev ${ }^{2 *}$ \\ ${ }^{1}$ Military-Technical Institute of the National Guard of the Republic of Uzbekistan, Tashkent \\ Uzbekistan \\ ${ }^{2}$ Tashkent State Transport University, Tashkent, Uzbekistan
}

\begin{abstract}
In this article, a mathematical model was developed to influence the intensity of the steering wheel turn by the driver on the vehicle's stability. Comparison of the developed mathematical model with the experiment results made it possible to establish their adequacy. The effect of the three conditional drivers on the intensity of the steering wheel was examined. When performing the 'J-turn' maneuver, comparing the indices of the 1st, 2nd and 3rd conditional drivers, their proximity to the requirements of the standard was established. It was found that the indices of the second conditional driver are closest to the standard requirements. When performing the 'Single Lane Change' maneuver by the first conventional driver, the standard deviation value from the specified trajectory was 0.102 , and the correlation index was 0.88 .
\end{abstract}

\section{Introduction}

Road traffic accidents occur mainly due to insufficient professional skills and work experience, improper maneuvering, and inattention to the speed limit. According to the results of the studies [4], it was determined that more than $50 \%$ of accidents as a result of car lane-change were committed due to non-compliance with the speed limit, improper maneuvering. More than $45 \%$ of accidents happened when overtaking vehicles due to improper maneuvering, a sharp lane change. When driving and making a maneuver, the driver takes the actions of varying degrees of intensity; this is especially important at the initial stage of the maneuver and at maintaining stready motion. To simulate the effect of the intensity of the impact on the steering wheel rotation, it is necessary to draw up equations that ensure sufficient adequacy to the process. A lot of research is being conducted in this direction [2, 3, 4]. Generally, equations of motion have the form of a system of higher-order equations, which pose some difficulties in solving them. The reason for this lies in need to determine the parameters of the vehicle units and parts based on the results of experimental studies. Hence, this article aims to develop a mathematical model of the influence of the intensity of the steering wheel rotation on the stability of the vehicle

\footnotetext{
* Corresponding author: davrstlei@gmail.com
} 
motion, convenient from all sides and revealing the process under study in sufficient volume.

\section{Materials and Methods}

The solution to the problem of modeling that provides the desired characteristics of controllability and stability of the vehicle movement requires drawing up a complete mathematical description, taking into account several degrees of freedom of the vehicle. An important condition in developing a mathematical description of a vehicle is to ensure the adequacy of modeling to a full-scale experiment. For this, the mathematical description for individual degrees of freedom must be sufficiently complete. The analysis of theoretical studies shows that to solve the problems of controllability and stability of vehicle movement, mathematical models of different completeness of description are used, including models that take into account two degrees of freedom of movement and multimass models that take into account 4-14 degrees of freedom [2-4].

A very important and most difficult link in the study of vehicle controllability and stability is the "driver". To date, various authors have proposed a mathematical description of the driver as a control link of the "driver-vehicle" system, taking into account his complex actions to maintain a given direction of movement. These models differ in the complexity and completeness of account for various factors characterizing the driver's behavior $[18,19]$.

As a result, it is advisable to ensure the vehicle's own stability and controllability, that is, its ability to resist external disturbances, execute control signals with the required accuracy and speed of operation when the driver is maintaining a given direction of movement. These modes of movement include the vehicle movement after an abrupt turn of the steering wheel - 'J-turn', "Single Lane Change", etc. For example, during the 'J-turn' tests, the driver's task is reduced to quickly turning the steering wheel at a certain angle, holding it in the rotated position until the end of the transient process, and maintaining the set speed. Therefore, this type of test is one of the main and is adopted in the standards [5, $6,16]$.

Besides, when performing a lane-changing maneuver, the intensity of the steering wheel is also considered as one of the input parameters of the vehicle maneuver.

Considering the above statements, a design scheme was developed for studying the curvilinear motion of a car (Fig. 1).

According to the design scheme, the car is presented as a two-axle vehicle, its center of the front wheel axle is at point $O_{1}$, and the center of the rear wheel axle is at point $O_{2}$.

For the study of curvilinear motion, two coordinate systems are selected, a fixed system $(x, y, z)$ and a movable system $\left(x_{m}, y_{m}, z_{m}\right)$.

The longitudinal axis of the machine is located at an angle $\psi$ concerning the x-axis. The steering angle of the front steering wheel is $\theta$.

The equation of motion of the car in the direction of the $Y$ axis can be written as follows:

$$
M_{a}\left(\dot{\psi}+\dot{\delta}_{a}\right) v_{a}-m_{n} h_{n} \ddot{\beta}=\sum Y_{1}+\sum Y_{2}
$$

where $M_{a}$ is the total mass of the vehicle, $\omega$ is the yaw rate of the vehicle's turn, $\delta_{a}$ is the vehicle slip angle (the angle between the velocity vector of the center of gravity of the vehicle and its longitudinal axis), $v_{a}$ is the vehicle speed, $m_{n}$ is the vehicle's sprung mass, $h_{r}$ 
is the distance from the center of gravity of the vehicle to the roll axis, $\beta$ is the roll angle of the body, $\Sigma Y_{f}$ and $\Sigma Y_{r}$ are the total lateral forces on the front and rear axles.
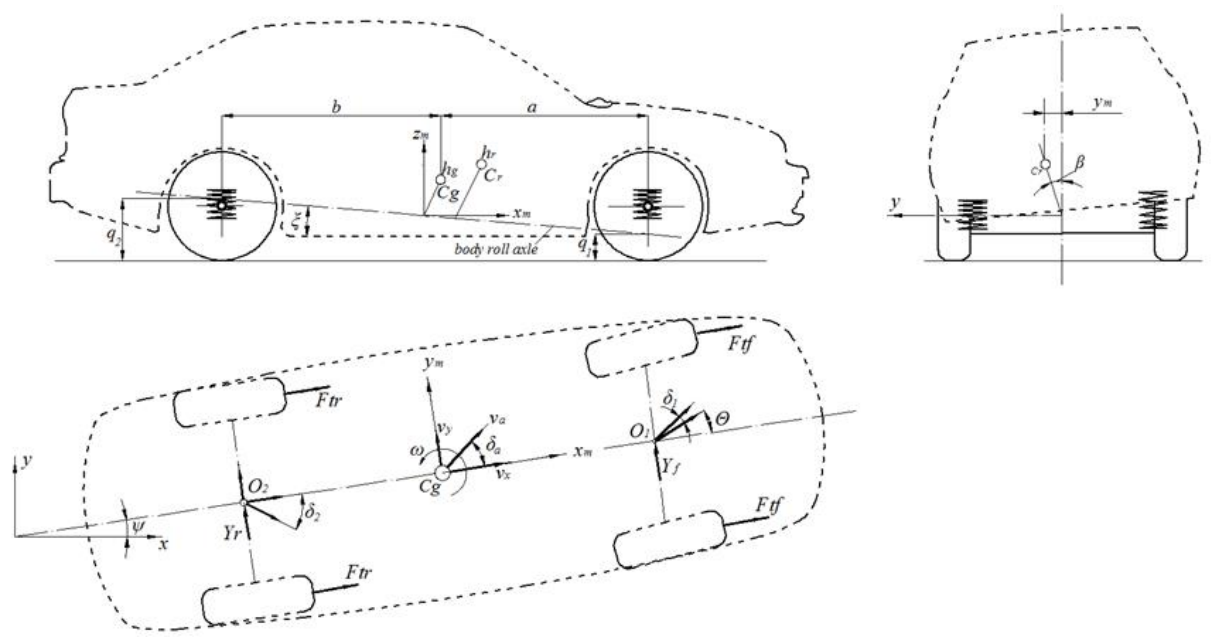

Fig. 1. Design scheme of vehicle movement

The relationship between slip angles and lateral forces is given as follows:

$$
Y_{i}=K_{y i} \delta_{i}
$$

where $K_{y i}$ is the coefficient of resistance to the slip of $i$-axes of the vehicle; $\delta$ is the angle of a slip of the axes.

The cornering coefficient of tire resistance can be represented as [8]:

$$
K_{y}=q_{Z} q_{T} K_{y 0}
$$

where $q_{Z}, q_{T}$ are the correction factors that take into account the effect of changes in vertical load, traction force and soil compressibility on the tire lateral slip; $K_{y 0}$ is the coefficient of resistance to a slip in the linear section of the dependence of the angle of slip on the lateral force.

The correction factor is determined by the following formula:

$$
q_{Z}=2.4 \frac{R_{z}}{G_{0}}-1.8\left(\frac{R_{z}}{G_{0}}\right)^{2}+0.4\left(\frac{R_{z}}{G_{0}}\right)^{3}
$$

where $R_{z}$ is the vertical load on the wheel; $G_{0}$ is the vertical load on the wheels corresponding to the maximum dependence on the slip resistance to lateral force.

To take into account the influence of traction force on the coefficient of slip resistance, the following formula is proposed [6]: 


$$
q_{T}=\sqrt{1-\left(\frac{P_{t i}}{R_{Z} \phi}\right)^{1.5}}
$$

where $P_{t i}$ is the traction force on the $i$-th wheel; $\varphi$ is the tire-to-surface friction coefficient (coefficient of adhesion).

The equation of motion of a vehicle relative to the $Z$ axis is:

$$
J_{z} \dot{\omega}+\left(J_{x z}-J_{z \xi}\right) \ddot{\beta}=\sum Y_{1} a-\sum Y_{2} b-\sum M_{c u}-\sum M_{c y}-\Delta M_{F}-M_{g}
$$

where $J_{z}$ is the moment of inertia of the vehicle wheel relative to the $Z$ axis, $I_{x z}$ is the centrifugal moment of inertia relative to the $x$ and $z$ axes, $I_{x \xi}$ is the moment of inertia of the sprung parts of the vehicle relative to the $x$ axis, $\Sigma M_{c u}$ is the total elastic stabilizing moment of tires; $\Sigma M_{c y}$ is the total stabilizing moment due to the longitudinal tilt of the king pin; $\Delta M_{F}$ is the moment from the difference in the forces of rolling resistance of the right and left wheels; $M_{g}$ is the gyroscopic moment that occurs when the camber variation changes; $a, b$ are the distances to the center of gravity of the vehicles from the center of the front and rear axles of the wheels $[22,23,24]$;

The equation of moments about the $X$-axis for the sprung masses is:

$$
J_{x n} \ddot{\beta}+J_{x z}(\dot{\omega}-\ddot{\beta} \xi)=m_{n} h_{r}\left(\omega+\dot{\delta}_{a}\right) v_{a}-\sum M_{c n}-\sum M_{\lambda} \pm M_{m p n}
$$

where $\sum M_{c n}, \sum M_{\lambda}, M_{m p n}$ represent the moments of resistance to the roll of the sprung masses of the elastic suspension elements, shock absorbers, and dry friction in the suspensions, respectively.

The equations of motion of the steered wheels relative to the pivot axis can be represented as follows:

$$
J_{K} \ddot{\Theta}=M_{c}+\mathrm{M}_{\lambda}+c_{p}\left(\frac{\alpha}{i}+\Theta\right)
$$

where $J_{z}$ is the moment of inertia of the car wheels, $M_{c}$ is the moment caused by the rolling of the tire slip and tire overhang, $M_{\lambda}$ is the viscous friction moment reduced to the axle of the steering wheel strut, $c_{p}$ is the steering gear stiffness, $\alpha$ is the steering wheel angle, $i$ is the steering gear ratio, $\Theta$ - steering wheel angle.

The equations of motion of the car considering (1), (7), (8), and (9) can be represented in the forms:

$$
\left\{\begin{array}{l}
M_{a}\left(\dot{\psi}+\dot{\delta}_{a}\right) v_{a}-m_{n} h_{r} \ddot{\beta}=\sum Y_{1}+\sum Y_{2} \\
J_{z} \dot{\omega}+\left(J_{x z}-J_{z \xi}\right) \ddot{\beta}=\sum Y_{1} a-\sum Y_{2} b-\sum M_{c u}-\sum M_{c y}-\Delta M_{F}-M_{g} \\
J_{x n} \ddot{\beta}+J_{x z}(\dot{\omega}-\ddot{\beta} \xi)=m_{n} h_{r}\left(\omega+\dot{\delta}_{a}\right) v_{a}-\sum M_{c n}-\sum M_{\lambda} \pm M_{m p n} \\
J_{K} \ddot{\Theta}=M_{c}+\mathrm{M}_{\lambda}+c_{p}\left(\frac{\alpha}{i}+\Theta\right)
\end{array}\right.
$$


When simulating the effect of the steering wheel intensity on the stability of the vehicle, the clearances and looseness in the steering system and steering wheel were not taken into account.

\section{Results and Discussion}

The purpose of the experimental research was to determine the transient and steady-state characteristics of the vehicle on the steering action and to obtain the necessary characteristics to test the adequacy of the mathematical model. The 'J-turn' and 'Single Lane Change' tests were selected to determine the transient response of the vehicle to driver input. This type of test is included in the interstate standard $[5,6,7,17,21]$, which regulates technical requirements for vehicles and test methods. The characteristics of the transient reactions of vehicles are normalized by an allowable range of transient and steadystate values for the steering ratio of the turn, i.e., the sensitivity of the vehicle to the steering action is normalized from the standpoint of vehicle controllability.

A measuring complex with special software was used for the experiments. The technical characteristics of the measuring complex are given in Table 1.

Table 1. The technical characteristics of the equipment

\begin{tabular}{|c|c|}
\hline \multicolumn{2}{|c|}{ Gyro sensors } \\
\hline Dynamic range & $\pm 450 \%$ \\
\hline Non-linearity in full range & $0.01 \%$ \\
\hline Mixing stability & $\pm 0035 \%$ \\
\hline \multicolumn{2}{|c|}{ Accelerometers } \\
\hline Dynamic range & $\pm 5 \mathrm{~g}$ \\
\hline Non-linearity in full range & $0.03 \%$ \\
\hline Rated power consumption & $1.3 \mathrm{~V}$. \\
\hline Temperature range & $-20 \div 70^{0} C$ \\
\hline
\end{tabular}

Figure $2 \mathrm{a}, \mathrm{b}$ shows the diagrams of performing 'J-turn' and "Single Lane Change" maneuvers by car drivers $[9,10,13,16]$.

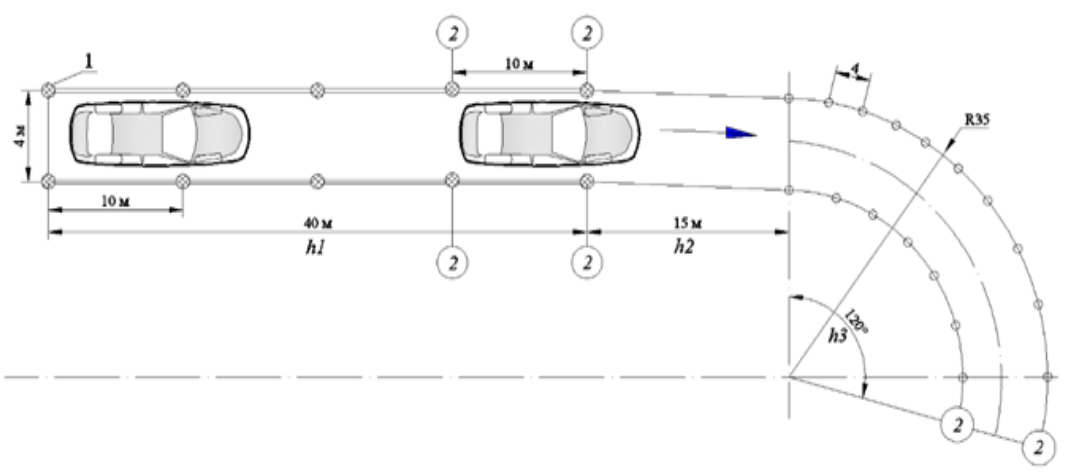

a) J-turn maneuver 


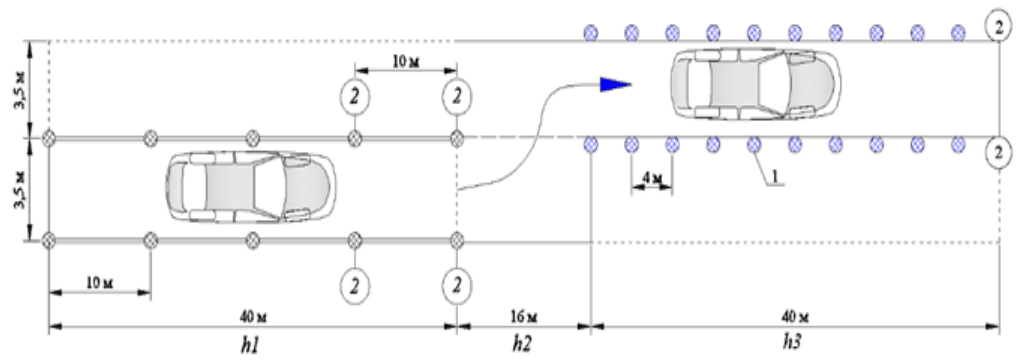

b) Single Lane Change maneuver

Fig. 2. Geometric dimensions of the roadway: 1 are vertical elements of the roadway; 2 are sensors for measuring the speed of motion; $h 1$ is acceleration element; $h 2$ is element of the beginning of the maneuver; $\mathrm{h} 3$ is interval to the end of the maneuver

Comparison of calculated and experimental data $[11,12,14,15]$. The experiments were conducted with various modes of vehicle motion. The experimental results are shown in Figures 3 and 4.

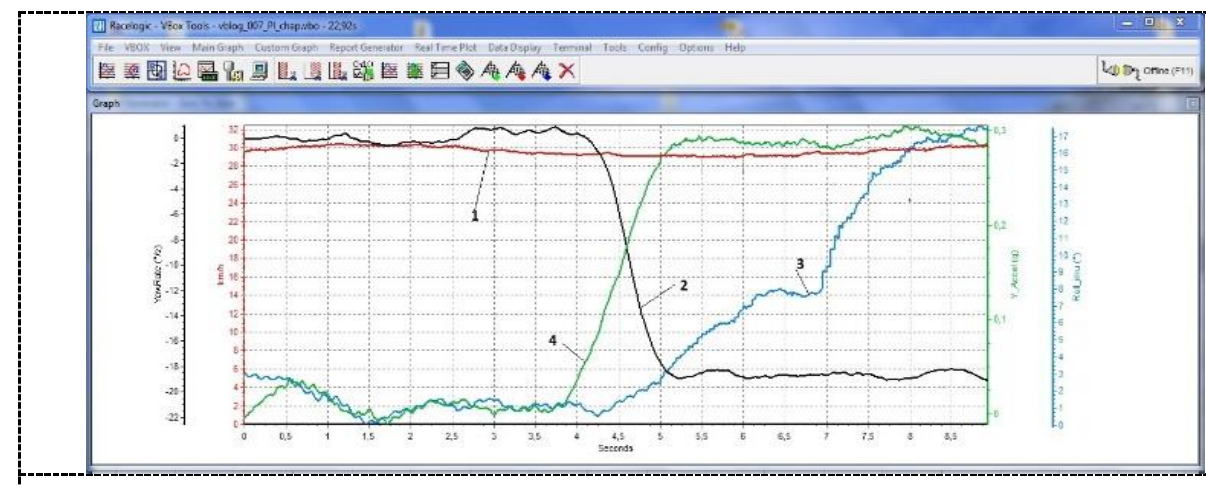

Fig. 3. 'J-turn' maneuver (turn to the left) 1 is vehicle speed; 2 is rotating speed; 3 is body roll; 4 is lateral acceleration

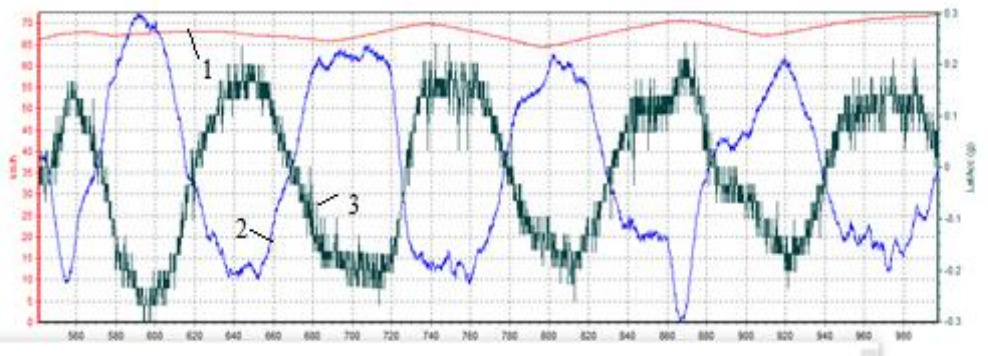

Fig. 4. 'Single Lane Change' maneuver 1 is vehicle speed; 2 is rotating speed; 3 is lateral acceleration

Determination of the degree of conformity of the vehicle characteristics, obtained by calculation with the experimentally obtained ones, was performed according to the transient characteristics of the angular rate of rotation of the vehicle at a sharp turn of the steering 
wheel. Figures 5 and 6 show the transient characteristics of the angular rate of rotation of the vehicle, obtained by calculation and experimental methods. The graphs show the calculated curves obtained in mathematical models that take into account $\left(\omega, v_{y}, \theta\right)-(\mathrm{I})$ and $\left(\omega, v_{y}, \beta, \theta\right)-(\mathrm{II})$.

The model taking into account $\left(\omega, v_{y}, \theta\right)$ gives satisfactory results at low values of transverse accelerations, not exceeding $1.0-2.0 \mathrm{~m} / \mathrm{s}^{2}$. With an increase in transverse accelerations and speed, the pattern of transient characteristics both qualitatively and quantitatively differs from the experimental ones.

The model taking into account $\left(\omega, v_{y}, \beta, \theta\right)$ gives qualitative and quantitative agreement with the experimental characteristics at transverse accelerations up to $4.0 \mathrm{~m} / \mathrm{s}^{2}$. The difference between the calculated and experimental characteristics in the speed range of $36-72 \mathrm{~km} / \mathrm{h}$ does not exceed $6-8 \%$. Thus, further computational studies were conducted using a mathematical model with three degrees of freedom.

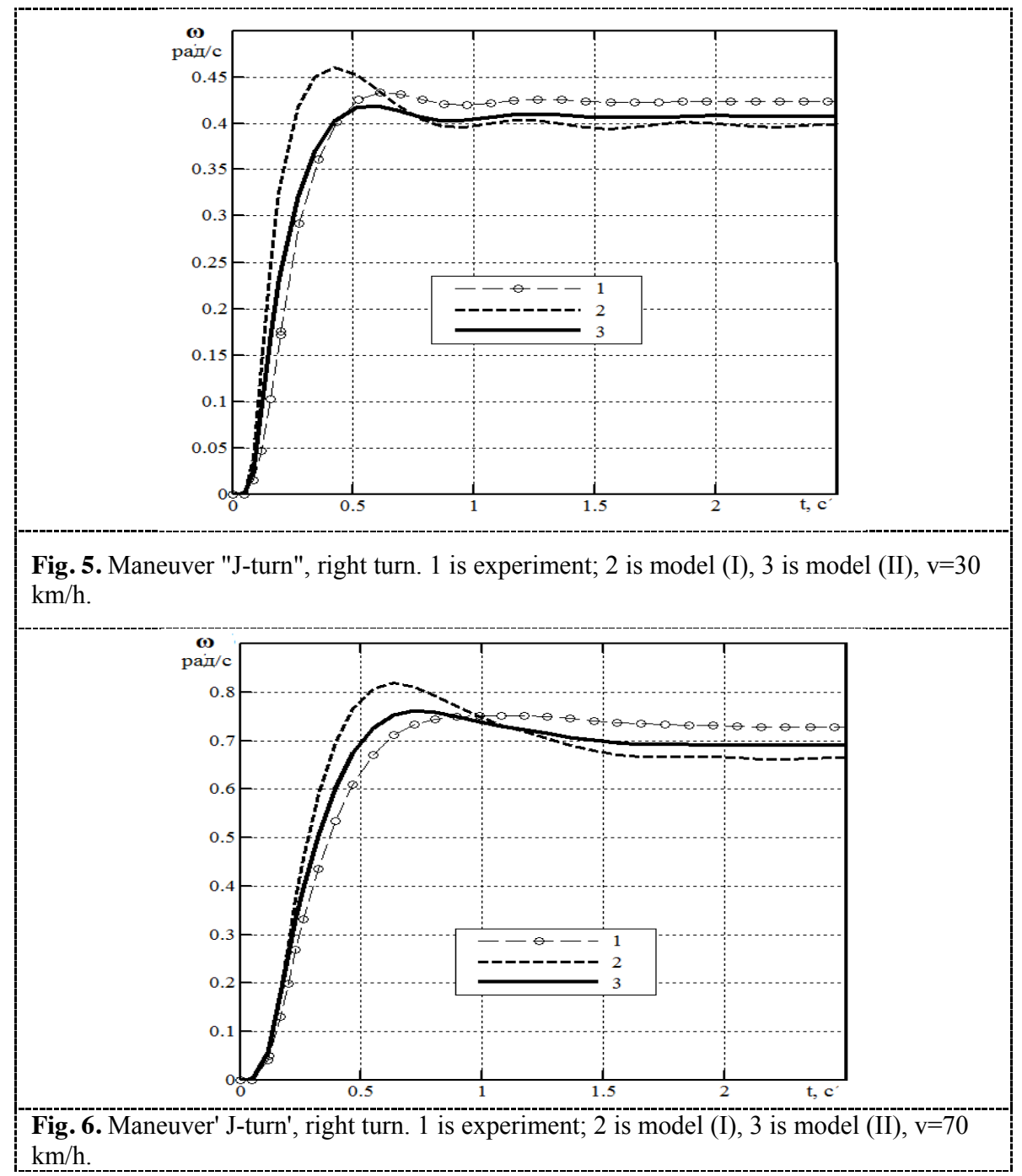




\subsection{Simulation results}

Computational modeling was carried out using the Matlab-Simulink program. Figs. 7 (a, b) show the intensity of the steering wheel rotation. When solving the equations of motion, the following values of the basic parameters of the car of category N1 were taken: $\mathrm{v}_{\mathrm{a}}=70 \mathrm{~km} / \mathrm{h}$; $\mathrm{M}_{\mathrm{a}}=1050 \mathrm{~kg} ; \mathrm{m}_{\mathrm{r}}=850 \mathrm{~kg} ; \mathrm{K}_{\mathrm{y} 01}=75000 \mathrm{~N} / \mathrm{rad} ; \mathrm{K}_{\mathrm{y} 02}=1.15 \mathrm{xK}_{\mathrm{y} 01}$ (understeer); hr=0.41 m; $\mathrm{J}_{\mathrm{Z}}=1820 \mathrm{kgm}^{2} ; \mathrm{a}=1.23 \mathrm{~m} ; \mathrm{b}=1.32 \mathrm{~m} ; \mathrm{J}_{\mathrm{K}}=8.8 \mathrm{kgm}^{2}$; the weight of conditional drivers $1,2,3$ $-75 \mathrm{~kg}$.

When performing the 'J-turn' maneuver to simulate the stability of the vehicle motion, the conditions for the steering wheel rotation at speed values $\alpha 1=300, \alpha 2=250, \alpha 3=200$ $\mathrm{rad} / \mathrm{s}$ were set to conditional drivers 1,2 and 3 .

After making a sharp turn of the steering wheel, the current value of the angular rate $\omega_{\text {cur }}$ of the vehicle rotation is held for 2-3 seconds until the steady value, i.e., until the value of $\omega_{s t a b}$, and then it is compared with the standard value.

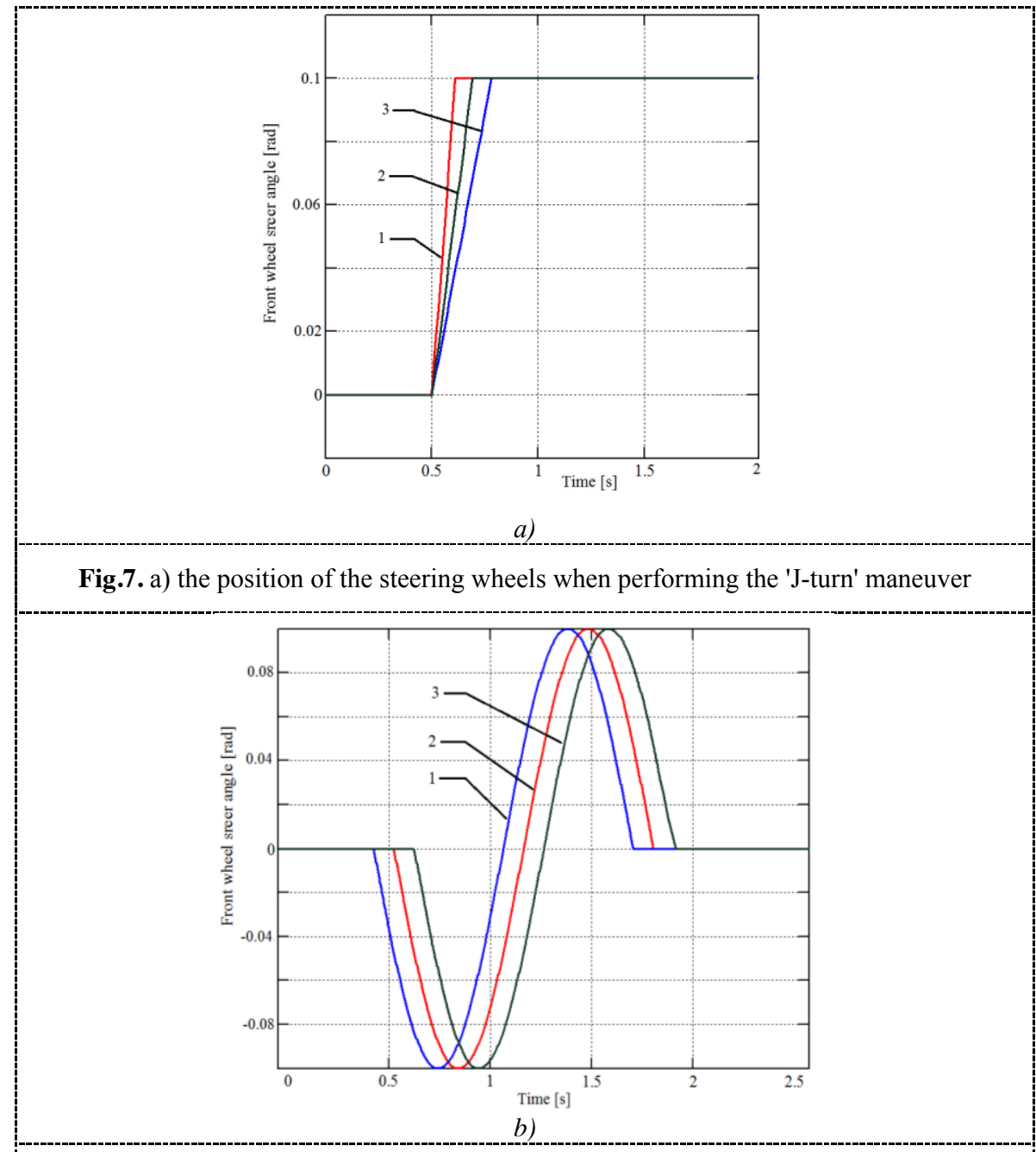

Fig. 7. a) the position of the steering wheels when performing the 'J-turn' maneuver; b) the position of the steering wheels when performing the 'Single Lane Change' maneuver. 
Table 2 shows the transient characteristics of the vehicle obtained at a speed of $70 \mathrm{~km} / \mathrm{h}$.

When the lateral acceleration $j_{y}=4 \mathrm{~m} / \mathrm{s}^{2}$ is reached, the car of category $\mathrm{N} 1$ should not exceed $20 \%$ of the reaction time of $0.3 \mathrm{~s}$ [5-7]. The relative overspeed of the angular velocity should not exceed $30 \%$.

When analyzing the indices of conventional drivers 1, 2 and 3 in terms of overspeed of the relative angular velocity, they are close to the standard ones, i.e., close to the indices for the highly qualified drivers. When analyzing $20 \%$ of the reaction time of drivers, only the second conditional driver had indices close to the standard ones.

Table 2. Experimental results

\begin{tabular}{|c|c|c|}
\hline $\begin{array}{c}\text { Conditional } \\
\text { drivers }\end{array}$ & $\begin{array}{c}\text { Relative overshoot angular } \\
\text { speed, } \%\left(\mathrm{j}_{\mathrm{y}}=4 \mathrm{~m} / \mathrm{s}^{2}\right)\end{array}$ & $\begin{array}{c}\text { Response time } 90 \%, \mathrm{~s} \\
\left(\mathrm{j}_{\mathrm{y}}=4 \mathrm{~m} / \mathrm{s}^{2}\right)\end{array}$ \\
\hline 1 & 26.2 & 0.45 \\
\hline 2 & 28.1 & 0.38 \\
\hline 3 & 13.3 & 0.59 \\
\hline
\end{tabular}

Standardized vehicle characteristics are shown in Fig. 7. The dotted line (R) shows the range of allowable values of the normalized transient response according to the standards.

Vehicle rated specifications are shown in Fig.7. In the same figure, the dotted line (R) shows the range of permissible values of the rated transient specifications according to the standard ones. As seen from Fig. 8 out of three conditional drivers, only the $3^{\text {rd }}$ driver goes beyond the established transient process; the indices of the first conditional driver, when entering the turn, at the beginning are stable, at an increase in the value of the angular rate of a vehicle rotation, the indices approach the extreme limits of the standard values.

The indices of the second conditional driver are within the standard values and show that at the end of the maneuver, the car motion is in a steady state.

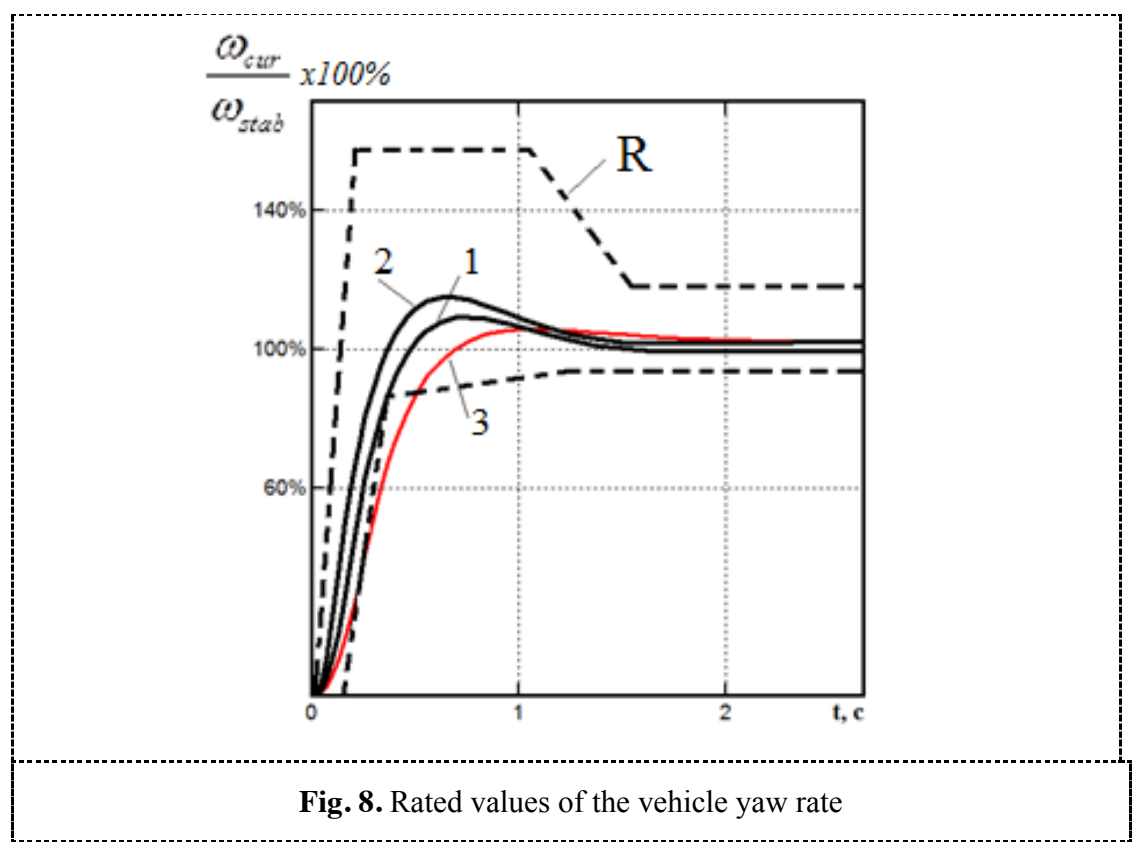

$\mathrm{R}$ is the range of allowable values of the rated transient response according to the standard 1, 2, and 3 conditional drivers, respectively.

To simulate the 'Single Lane Change' maneuver, the middle line of the R road corridor is set as: 


$$
\begin{gathered}
y_{0}=y_{0}^{1}+y_{0}^{2}, \\
y_{0}^{1}= \begin{cases}\alpha v_{a} t, \quad \text { at } \mathrm{t}>0 \\
0, \quad \text { at } \mathrm{t}<0\end{cases} \\
y_{0}^{2}= \begin{cases}-\alpha v_{a}\left(t-\frac{l}{v_{a}}\right), & \text { at } \mathrm{t}>\frac{l}{v_{a}} \\
0, & \text { at } \mathrm{t} \prec \frac{l}{v_{a}}\end{cases}
\end{gathered}
$$

When simulating a maneuver for conditional drivers 1, 2 and 3, the following values of the steering wheel turn intensity are entered: $\alpha_{1}=120, \alpha_{2}=140, \alpha_{3}=200 \mathrm{rad} / \mathrm{s}$, respectively, the speed is $70 \mathrm{~km} / \mathrm{h}$. The results of the 'Single Lane Change' maneuver simulation are shown in Fig. 9.

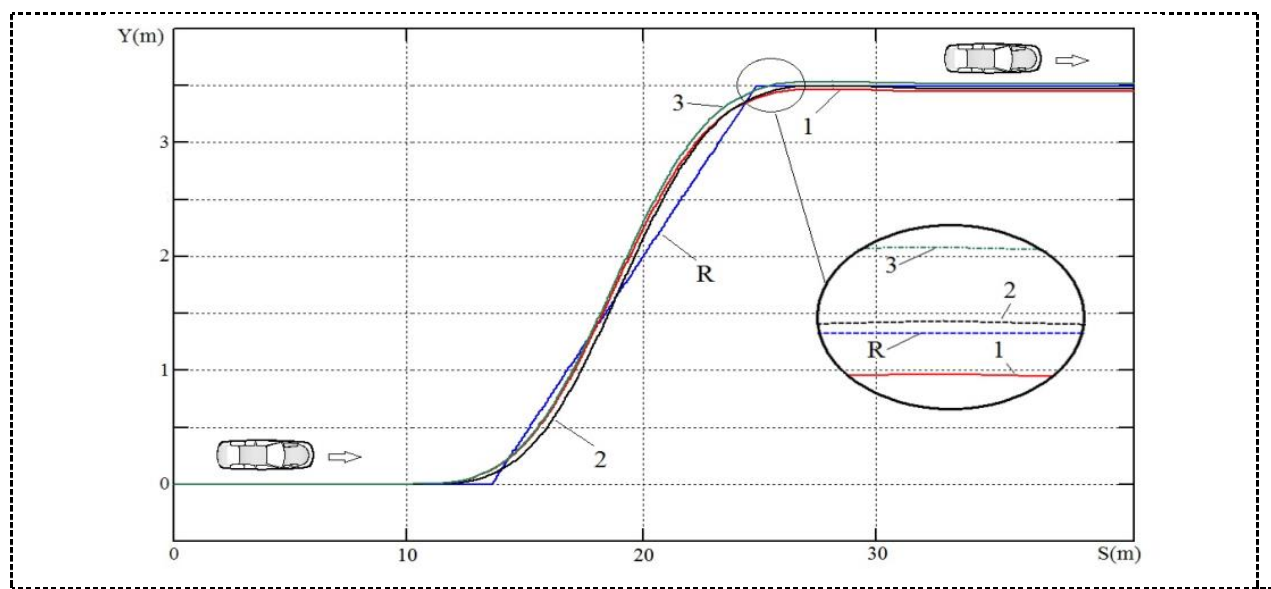

Fig. 9. 'Single Lane Change' maneuver. Desired (R) and realized trajectories $(1,2,3)$ of vehicle maneuvers

As seen from Fig. 9, conditional drivers make maneuvers along different trajectories, depending on the intensity of the steering wheel rotation.

To determine the standard deviation $E_{r(y)}^{2}$ from a given trajectory, the following formula is used:

$$
E_{r(y)}^{2}=\sum\left(d_{i}-R\right)^{2}
$$

where $d_{i}$ are the $i$ coordinates of the driver deviation values $(i=1,2,3)$,

$R$ is the given coordinate of the road.

In formula (14), the less the values of $E_{r(y)}^{2}$, the less the error in the calculation results. 


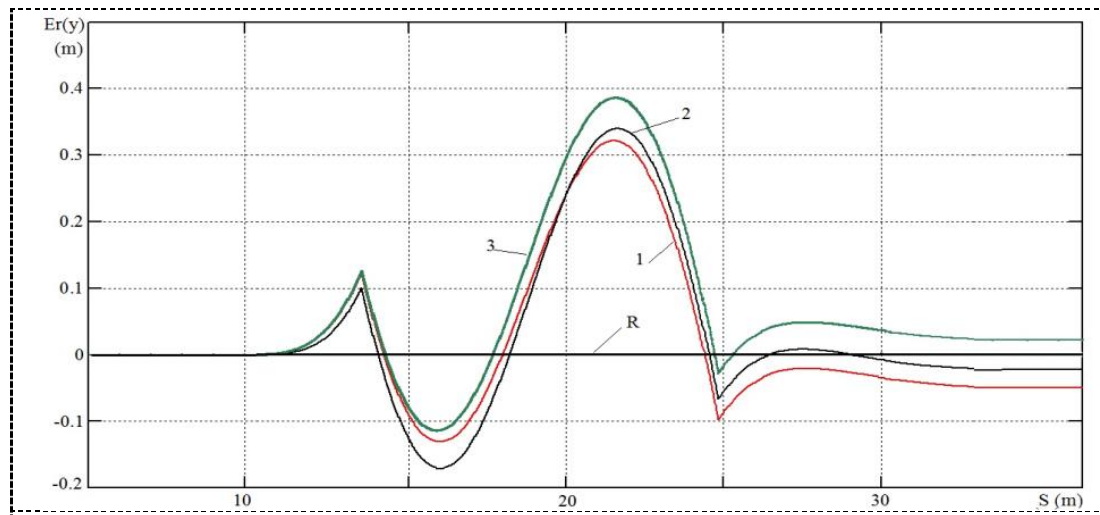

Fig. 10. 'Single Lane Change' maneuver. Deviation of the vehicle trajectory from the set one $(\mathrm{R})$, controlled by different drivers $(1,2,3)$

There is an index that characterizes the tightness of the $d_{i}$ and $R$ constraint, called the correlation coefficient $k$ and calculated by the formula:

$$
k=\sqrt{1-\frac{\sum_{i=1}^{n}\left(y_{i}-R_{i}\right)^{2}}{\sum_{i=1}^{n}\left(y_{i}-M_{y}\right)^{2}}}
$$

where $y_{i}$ is the value of the realized trajectory of the $i$-th driver, (R) is the value of the desired trajectory, and $M_{y}$ is the average value of $y$.

The correlation index in its absolute value ranges from 0 to 1 . At low deviations, the correlation index $k$ is closer to 1 .

Table 3. The simulation results are shown.

\begin{tabular}{|c|c|c|}
\hline Conditional drivers & $E_{r(y)}^{2}$ & $K$ \\
\hline 1 & 0.102 & 0.88 \\
\hline 2 & 0.115 & 0.76 \\
\hline 3 & 0.144 & 0.69 \\
\hline
\end{tabular}

As seen from Table 3 and Figure 10, the first conditional driver has the least standard deviation and the correlation index -0.102 and 0.88 , respectively.

\section{Conclusions}

Based on the results of theoretical and experimental studies of two types of maneuvers, it was found that the intensity of the steering wheel rotation has a different effect on the motion stability of the vehicle.

For instance, when analyzing the conditional driver in the 'J-turn' maneuver, the performance of 1,2,3 conditional drivers is close to the standard as a qualified driver. If we analyze taking into account reaction time for $90 \%$, only the second conditional driver comes to close to the standard. In the "Single Lane Change" maneuver, the conditional 1st driver has a correlation index of 0.88 , corresponding to a minimum square deviation of 0.102 .

The developed modeling method makes it possible to predict stability in the 'drivervehicle' system. 


\section{References}

1. Borisov B.I. On the classification of the causes of road traffic accidents, Vestnik SSTU, 2 (71), pp. 366-369. (2013).

2. S. Ikehaga, Active Suspension Control of Ground Vehicle based on a Full-Vehicle Model, American Control Conference, pp. 4019-4024, (2000).

3. Guo L, Ge PS, Yue M, et al. Lane changing trajectory planning and tracking controller design for intelligent vehicle running on curved road, Math Probl Eng 2014, pp.1-9, (2014)

4. Yoshida H, Shinohara S and Nagai M. Lane change steering maneuvers using model predictive control theory. Veh Syst Dynam, 46, pp. 669-681. (2008).

5. Motor vehicles. Controllability and stability. Test methods. Interstate standards. GOST 31507-2012. M.: Standart inform, (2013)

6. ISO 3888-1:1999 Passenger cars - Test track for a severe lane-change manoeuvre - Part 1: Double line-change. (1999)

7. ISO 3888-2:2002 Passenger cars - Test track for a severe lane-change manoeuvre - Part 2: Obstacle avoidance, (2000)

8. Antonov D.A. The theory of stability of motion of multi-axle vehicles. M.: Mashinostroenie, p. 216, (1987)

9. Allen, R.W., Christos, J.P, 2002, "Driver/Vehicle Modeling and Simulation", SAE Transations, 2002-01-1568. (2002)

10. Allen, R.W., Rosenthal, T.J. Computer Simulation Analysis of Light Vehicle Lateral, Directional Dynamic Stability, SAE Transactions Paper No. 1999-010124, (1999)

11. Bernard, J., Gruening, J., and Hoffmeister, K., Evaluation of Vehicle,Driver Performance Using Genetic Algoritms, SAE Transactions Paper, 980227.(1998).

12. U.S. Department of Transportation NHTSA: Traffic Safety Facts 2002. Washington DC, (2004)

13. Flystra, D., Lasdon, L., Watson, J. and Waren, A, "Design and Use of the Microsoft Excel Solver", Interfaces, 28, (5), pp. 29-55, (1998)

14. Fraichard, T., and Scheuer, A., From Reeds and Shepp's to Continuous-Curvature Paths, IEEE Transactions on Robotics, 20, (6), pp. 1025-1035. (2004)

15. Forkenbrock, G.J., Garrott, W.R., Light Vehicle Dynamic Rollover Propensity Phases IV, (V), and (VI), Research Activities", Unpublished NHTSA presentation (http://www-nrd.nhtsa.dot.gov/pdf/nrd-01/SAE/SAE2002/RGarrott rollover.pdf). (2002)

16. Forkenbrock, G.J., Heitz, M., etc, An Experimmental Examination of J-Turn and Fishhook Maneuvers That May Induce On-Road, Untripped, Light Vehicle Rollover, SAE Transactions Paper No. 2003-01-1008, (2003)

17. Forkenbrock, G.J., Heitz, M, etc., An Experimmental Examination of Double Lane Change Maneuvers That May Induce On-Road, Untripped, Light Vehicle Rollover", SAE Transactions Paper No. 2003-01-1009, (2003)

18. Forkenbrock, G.J., O'Harra, B.C., Elsasser D. An Experimental Examination of 26 Light Vehicles Using Test Maneuvers That May Induce On-Road, Untripped Rollover and a Discussion of NHTSAs Refined Test Procedures -Phases VI and VII of NHTSAs Light Vehicle Rollover Research Program, U.S. Department of Transportation Report No. DOT HS 809 547, (2003)

19. Frimberger, M., Wolf, F., Scholpp, G. etc., Influences of Parameters at Vehicle Rollover, SAE Transactions Paper No. 2000-01-2669, (2000)

20. Gillespie, T.D. Fundamentals of Vehicle Dynamics, Society of Automotive Engineers, Warrendale, PA.(1992) 
21. Langer, W., Validation of Flat Surface Roadway Technology, Society of Automotive Engineers Paper No. 950310, (1995)

22. Langer, W., Vehicle Testing With Flat Surface Roadway Technology, Society of Automotive Engineers Paper No. 960731, (1996)

23. Lasdon, L.S. and Smith, S., Solving large sparse nonlinear programs using GRG, ORSA Journal on Computing, 4,(1), pp. 2-15, (1992)

24. Marine, M.C., Wirth, J.L. and Thomas, T.M., Characteristics of On-Road Rollovers, SAE Transactions Paper No. 1999-01-0122, (1999) 